\title{
On a special type of solutions of arbitrary higher spin Dirac operators
}

\author{
H. De Schepper ${ }^{\dagger}$, D. Eelbode*, T. Raeymaekers ${ }^{\dagger}$ \\ * Department of Mathematics and Computer Science, University of Antwerp \\ Campus Middelheim, G-Building, Middelheimlaan 1, 2020 Antwerpen, Belgium \\ ${ }^{\dagger}$ Clifford research group, Department of Mathematical Analysis, Ghent University \\ Galglaan 2, 9000 Ghent, Belgium \\ E-mail: hds@cage.ugent.be, david.eelbode@ua.ac.be, tr@cage.ugent.be
}

\begin{abstract}
In this paper an explicit expression is determined for the elliptic higher spin Dirac operator, acting on functions $f(\underline{x})$ taking values in an arbitrary irreducible finite-dimensional module for the group $\operatorname{Spin}(m)$ characterized by a half-integer highest weight. Also a special class of solutions of these operators is constructed, and the connection between these solutions and transvector algebras is explained.
\end{abstract}

PACS numbers: $2.20 \mathrm{Qs}, 2.30 \mathrm{Px}$

Submitted to: J. Phys. A: Math. Gen. 


\section{Introduction}

Classical Clifford analysis is usually defined as a function theory generalizing complex analysis to the case of arbitrary dimension $m \in \mathbb{N}$ (considered as a formal parameter), and at the same time refining classical harmonic analysis in $\mathbb{R}^{m}$. The main object of study in this theory is the so-called Dirac operator, which generalizes the CauchyRiemann operator and factorizes the Laplacian $\Delta_{m}$. We refer the reader to $[1,2,3]$ or to the overview paper [4] for a general introduction to this branch of classical analysis. From a more algebraic point of view, the Dirac operator can be defined as the unique (up to a multiplicative constant) first-order differential operator acting on spinor-valued functions, which is spin-invariant. To be more precise: this operator is in fact conformally invariant, a property which is usually described in terms of Vahlen matrices in Clifford analysis, but for most purposes it suffices to consider the rotational invariance, w.r.t. a suitable action of the spin group. Within the setting of conformal (or Riemannian) geometry the construction of such (invariant) operators is well-known, see e.g. [5, 6, 7], and can be established using the method of generalized gradients. It should however be stressed that the questions addressed in Clifford analysis are of a completely different nature: one is mainly interested in the space of polynomial solutions, integral representations for arbitrary solutions, integral transforms, etc... Since a few years, Clifford analysis has turned out to be a very elegant framework to study this type of problems not only for the classical Dirac operator, but also for far-reaching generalizations of it acting on functions which take their values in arbitrary half-integer irreducible spinrepresentations. The earliest generalizations involved the so-called Rarita-Schwinger operator, again inspired by equations coming from theoretical physics; in $[8,9]$ one can find the function theoretical fundaments for spin-invariant operators acting on functions taking values in irreducible modules with highest weight $\left(k+\frac{1}{2}, \frac{1}{2}, \cdots, \frac{1}{2}\right)$. The present paper is part of a program to establish and study the most general possible operator (and its solutions), in which both the dimension and the highest weight vector of the underlying value space are treated as parameters: this is the subject of Clifford analysis for higher spin Dirac operators.

This paper is organized as follows: after an introduction to the language of Clifford analysis in section 2, we establish the general higher spin Dirac operator in section 3 , as a first-order differential operator acting on functions in several vector variables. Subsequently, we introduce a special class of solutions in section 4 and investigate their connection with transvector algebras in section 5 .

\section{Clifford Analysis}

Let $\left(\underline{e}_{1}, \ldots, \underline{e}_{m}\right)$ be an orthonormal basis for the Euclidean vector space $\mathbb{R}^{m}$, and denote by $\mathbb{R}_{m}$ the real Clifford algebra generated by these basis elements, together with the defining relations $\underline{e}_{i} \underline{e}_{j}+\underline{e}_{j} \underline{e}_{i}=-2 \delta_{i j}, i, j=1, \ldots, m$. The complex Clifford algebra $\mathbb{C}_{m}$ is then defined as $\mathbb{C}_{m}:=\mathbb{R}_{m} \otimes_{\mathbb{R}} \mathbb{C}$. Any element $\underline{x}$ of $\mathbb{R}^{m}$ can be embedded inside the 
Clifford algebra, by means of $\underline{x} \hookrightarrow \sum \underline{e}_{j} x_{j}$. The scalar valued Euclidian inner product can then be defined as

$$
\langle\underline{x}, \underline{y}\rangle=-\frac{1}{2}(\underline{x} \underline{y}+\underline{y} \underline{x})=\sum_{j=1}^{m} x_{j} y_{j}
$$

Inside the complex Clifford algebra $\mathbb{C}_{m}$, one can define the spinor space(s) $\mathbb{S}^{ \pm}$as a minimal left ideal, using a primitive idempotent. Note that the parity of spinors only needs to be taken into account in case of even dimension $m=2 n$. From now on we will often omit this reference to the parity of the spinors, which amounts to saying that we will be mostly dealing with odd dimensions $m=2 n+1$. However, up to a minor addition of \pm signs, our results will also hold for the case of even dimensions: this will be mentioned whenever it is needed. The vector space $\mathbb{S}$ defines the basic half-integer representation for the spin group $\operatorname{Spin}(m)$, described by the highest weight $\left(\frac{1}{2}, \frac{1}{2}, \cdots, \frac{1}{2}\right)$ under the action $\psi \mapsto s \psi$ for all $\psi \in \mathbb{S}$ and $s \in \operatorname{Spin}(m)$. Note that the spin group can itself be realized inside the Clifford algebra by

$$
\operatorname{Spin}(m)=\left\{s=\prod_{j=1}^{2 k} s_{j}: k \in \mathbb{N}, s_{j} \in S^{m-1}\right\}
$$

where $S^{m-1} \subset \mathbb{R}^{m}$ denotes the unit sphere in $\mathbb{R}^{m}$. The classical Dirac operator in $\mathbb{R}^{m}$ is given by $\underline{\partial}_{x}=\sum_{j} \underline{e}_{j} \partial_{x_{j}}$. This is the unique spin-invariant first order differential operator acting on $\mathbb{S}$-valued functions $f(\underline{x})$ on $\mathbb{R}^{m}$, with respect to the regular representation $f(\underline{x}) \mapsto s f(\bar{s} \underline{x} s)$. We call such an $\mathbb{S}$-valued function monogenic in $\underline{x}$ if it satisfies $\underline{\partial}_{x} f=0$. The Dirac operator $\underline{\partial}_{x}$ and the vector variable $\underline{x}$ generate the Lie superalgebra osp $(1,2)$, which is a concise way to summarize the most crucial operator identities in Clifford analysis. We e.g. have the following relation, which will be used later:

$$
\left\{\underline{x}, \underline{\partial}_{x}\right\}:=\underline{x}_{x}+\underline{\partial}_{x} \underline{x}=-2\left(\mathbb{E}_{x}+\frac{m}{2}\right)
$$

where $\mathbb{E}_{x}=\sum_{j} x_{j} \partial_{x_{j}}$ is the Euler operator on $\mathbb{R}^{m}$.

It is crucial to observe that not only the spinor space $\mathbb{S}$, but also other half-integer $\operatorname{Spin}(m)$-representations can be characterized in the language of Clifford algebras and Clifford analysis, see e.g. [10]. This is done using the notion of Clifford analysis in several vector variables $\underline{u}_{i} \in \mathbb{R}^{m}$. From now on, we will denote $\underline{\partial}_{u_{i}}$ by $\underline{\partial}_{i}$.

Definition 1 A function $f: \mathbb{R}^{k m} \rightarrow \mathbb{C}:\left(\underline{u}_{1}, \ldots, \underline{u}_{k}\right) \mapsto f\left(\underline{u}_{1}, \ldots, \underline{u}_{k}\right)$ is called simplicial harmonic if it satisfies the system

$$
\begin{aligned}
& \left\langle\underline{\partial}_{i}, \underline{\partial}_{j}\right\rangle f=0, \text { for all } i, j=1, \ldots, k \\
& \left\langle\underline{u}_{i}, \underline{\partial}_{j}\right\rangle f=0, \text { for all } 1 \leq i<j \leq k
\end{aligned}
$$

The vector space of $\mathbb{C}$-valued simplicial harmonic polynomials which are $l_{i}$-homogeneous in $\underline{u}_{i}$ will be denoted by $\mathcal{H}_{l_{1}, \ldots, l_{k}}$, where, from now on, we assume $l_{1} \geq \cdots \geq l_{k}$. 
Definition 2 A function $f: \mathbb{R}^{k m} \rightarrow \mathbb{S}:\left(\underline{u}_{1}, \ldots, \underline{u}_{k}\right) \mapsto f\left(\underline{u}_{1}, \ldots, \underline{u}_{k}\right)$ is called simplicial monogenic if it satisfies the system

$$
\begin{aligned}
& \underline{\partial}_{i} f \quad=0, \text { for all } i=1, \ldots, k \\
& \left\langle\underline{u}_{i}, \underline{\partial}_{j}\right\rangle f=0, \text { for all } 1 \leq i<j \leq k
\end{aligned}
$$

The vector space of $\mathbb{S}$-valued simplicial monogenic polynomials which are $l_{i}$-homogeneous in $\underline{u}_{i}$ will be denoted by $\mathcal{S}_{l_{1}, \ldots, l_{k}}$, where again, from now on, we assume that $l_{1} \geq \cdots \geq l_{k}$. The following definition involves weaker conditions on the $\mathbb{S}$-valued functions, but will nevertheless be crucial in what follows.

Definition 3 A function $f: \mathbb{R}^{k m} \rightarrow \mathbb{S}:\left(\underline{u}_{1}, \ldots, \underline{u}_{k}\right) \mapsto f\left(\underline{u}_{1}, \ldots, \underline{u}_{k}\right)$ is called monogenic if it satisfies $\underline{\partial}_{i} f=0$, for all $1 \leq i \leq k$.

The vector space of $\mathbb{S}$-valued monogenic polynomials which are $l_{i}$-homogeneous in $\underline{u}_{i}$ will be denoted by $\mathcal{M}_{l_{1}, \ldots, l_{k}}$, again with $l_{1} \geq \cdots \geq l_{k}$. Each of these polynomial vector spaces can be seen as a module for the spin group, under the regular representation (or so-called $L$-representation) given by

$$
L(s) P\left(\underline{x}_{1}, \ldots, \underline{x}_{k}\right):=s P\left(\bar{s} \underline{x}_{1} s, \ldots, \bar{s} \underline{x}_{k} s\right), \quad s \in \operatorname{Spin}(m)
$$

In e.g. [10], it was proven that under this action, the $\operatorname{Spin}(m)$-modules $\mathcal{H}_{l_{1}, \cdots, l_{k}}$ (resp. $\mathcal{S}_{l_{1}, \cdots, l_{k}}$ ) define a model for the irreducible highest weight representation characterized by means of

$$
\begin{aligned}
& \mathcal{H}_{l_{1}, \cdots, l_{k}} \rightarrow\left(l_{1}, \cdots, l_{k}, 0, \cdots, 0\right):=\left(l_{1}, \cdots, l_{k}\right) \\
& \mathcal{S}_{l_{1}, \cdots, l_{k}} \rightarrow\left(l_{1}+\frac{1}{2}, \cdots, l_{k}+\frac{1}{2}, \frac{1}{2}, \cdots, \frac{1}{2}\right):=\left(l_{1}, \cdots, l_{k}\right)^{\prime}
\end{aligned}
$$

As shown in the above notations, we will omit redundant zeros in the highest weight vector, and denote the Cartan product by means of a prime. Note that in case $m=2 n$, one should also add a parity index to the spaces of simplicial monogenics.

\section{Construction of higher spin Dirac operators}

The aim of this section is to find an explicit expression for the $\operatorname{Spin}(m)$-invariant differential operator

$$
\begin{aligned}
\mathcal{Q}_{l_{1}, \ldots, l_{k}}: \mathcal{C}^{1}\left(\mathbb{R}^{m}, \mathcal{S}_{l_{1}, \ldots, l_{k}}\right) & \rightarrow \mathcal{C}^{0}\left(\mathbb{R}^{m}, \mathcal{S}_{l_{1}, \ldots, l_{k}}\right) \\
f\left(\underline{x} ; \underline{u}_{1}, \ldots, \underline{u}_{k}\right) & \mapsto \mathcal{Q}_{l_{1}, \ldots, l_{k}} f\left(\underline{x} ; \underline{u}_{1}, \ldots, \underline{u}_{k}\right)
\end{aligned}
$$

which will be our most general higher spin Dirac operator. Note that in the case where $m=2 n$, these operators change the parity of the spinors (so called parity-reversion):

$$
\mathcal{Q}_{l_{1}, \ldots, l_{k}}: \mathcal{C}^{1}\left(\mathbb{R}^{m}, \mathcal{S}_{l_{1}, \ldots, l_{k}}^{ \pm}\right) \rightarrow \mathcal{C}^{0}\left(\mathbb{R}^{m}, \mathcal{S}_{l_{1}, \ldots, l_{k}}^{\mp}\right)
$$

The existence and uniqueness of such an invariant differential operator is guaranteed because of Fegan's result [6] on conformally invariant operators. As we are mainly 
focusing on rotational invariance in this paper, we can also invoke the Stein-Weiss paper [7] on generalized gradients to explain this: it suffices to note that for any highest weight module $\left(l_{1}, \cdots, l_{k}\right)^{\prime}$ the tensor product with the standard representation $\mathbb{C}^{m}$ contains the module $\left(l_{1}, \cdots, l_{k}\right)^{\prime}$ as a summand. In abstract terms, we thus have:

$$
\mathcal{C}^{1}\left(\mathbb{R}^{m},\left(l_{1}, \cdots, l_{k}\right)^{\prime}\right) \stackrel{\nabla}{\longrightarrow} \mathcal{C}^{0}\left(\mathbb{R}^{m}, \mathbb{C}^{m} \otimes\left(l_{1}, \cdots, l_{k}\right)^{\prime}\right) \stackrel{\pi}{\longrightarrow} \mathcal{C}^{0}\left(\mathbb{R}^{m},\left(l_{1}, \cdots, l_{k}\right)^{\prime}\right)
$$

with $\pi$ a suitable projection operator. Note that we put a limit on the number of variables in terms of the dimension $m=2 n+1$ ( or $m=2 n$ ): we will always assume that $k<n$. The case $k=n$ is special, and leads to generalizations of the massless-field operator, whereas the case $k>n$ is often referred to in the literature as the non-stable range. In the case $k=2$, we will reobtain the operator $\mathcal{Q}_{l_{1}, l_{2}}$ introduced in $[11,12]$. There, it was found that (up to a multiplicative constant)

$$
\mathcal{Q}_{l_{1}, l_{2}}=\left(1+\frac{\underline{u}_{1} \underline{\partial}_{1}}{m+2 l_{1}-2}\right)\left(1+\frac{\underline{u}_{2} \underline{\partial}_{2}}{m+2 l_{2}-4}\right) \underline{\partial}_{x}
$$

In the case $k=1$, the operator reduces to

$$
\mathcal{Q}_{l_{1}}=\left(1+\frac{\underline{u}_{1} \underline{\partial}_{1}}{m+2 l_{1}-2}\right) \underline{\partial}_{x}
$$

This operator is known as the Rarita-Schwinger operator $\mathcal{R}_{l_{1}}$, see e.g. $[8,9]$. We will now establish the general form of the higher spin Dirac operator by construction.

Theorem 1 Up to a multiplicative constant the higher spin Dirac operator

$$
\mathcal{Q}_{l_{1}, \ldots, l_{k}}: \mathcal{C}^{1}\left(\mathbb{R}^{m}, \mathcal{S}_{l_{1}, \ldots, l_{k}}\right) \rightarrow \mathcal{C}^{0}\left(\mathbb{R}^{m}, \mathcal{S}_{l_{1}, \ldots, l_{k}}\right), f(\underline{x}) \mapsto \mathcal{Q}_{l_{1}, \ldots, l_{k}} f(\underline{x})
$$

takes the form

$$
\mathcal{Q}_{l_{1}, \ldots, l_{k}}=\left(\prod_{i=1}^{k}\left(1+c_{i} \underline{u}_{i} \underline{\partial}_{i}\right)\right) \underline{\partial}_{x}
$$

where the constants $c_{i}$ are explicitly given by $c_{i}=\frac{1}{m+2 l_{i}-2 i}$.

Proof.

We need to show the existence of a nonzero set of constants $c_{i}$, such that, when applying the operator given by $(1)$, it holds for all $f$ in $\mathcal{C}^{\infty}\left(\mathbb{R}^{m}, \mathcal{S}_{l_{1}, \ldots, l_{k}}\right)$ that

$$
\underline{\partial}_{1} \mathcal{Q}_{l_{1}, \ldots, l_{k}} f=0
$$

and

$$
\left\langle\underline{u}_{i}, \underline{\partial}_{i+1}\right\rangle \mathcal{Q}_{l_{1}, \ldots, l_{k}} f=0, \quad i=1, \ldots, k-1
$$

Indeed, since $\underline{\partial}_{i+1}=\left[\underline{\partial}_{i},\left\langle\underline{u}_{i}, \underline{\partial}_{i+1}\right\rangle\right]$, all other conditions contained in Definition 2 will follow from the above ones. We thus only have $k$ conditions to impose, which corresponds to the fact that there are $k$ constants to be determined in the expression for $\mathcal{Q}_{l_{1}, \ldots, l_{k}}$. Using standard operator identities from Clifford analysis, it follows from the condition

$$
\underline{\partial}_{1} \prod_{i=1}^{k}\left(1+c_{i} \underline{u}_{i} \underline{\partial}_{i}\right) \underline{\partial}_{x} f=0
$$


that $c_{1}=\left(m+2 k_{1}-2\right)^{-1}$, where we have used the fact that $f$ is $\mathcal{S}_{l_{1}, \ldots, l_{k}}$-valued. Similar calculations show that from the condition

$$
\left\langle\underline{u}_{i}, \underline{\partial}_{i+1}\right\rangle \prod_{j=1}^{k}\left(1+c_{j} \underline{u}_{j} \underline{\partial}_{j}\right) \underline{\partial}_{x} f=0, \quad i \in\{1, \ldots, k-1\}
$$

it follows that $c_{i+1}=c_{i}\left(1+2 c_{i}\left(l_{i+1}-l_{i}-1\right)^{-1}\right)$, for all $f \in \mathcal{C}^{\infty}\left(\mathbb{R}^{m}, \mathcal{S}_{l_{1}, \ldots, l_{k}}\right)$. Inductively we thus arrive at $c_{i}=\left(m+2 l_{i}-2 i\right)^{-1}$, as had to be proven.

Note that one can also rewrite this operator without explicitly mentioning the precise form of the highest weight fixing our values. Indeed, it suffices to replace each constant $c_{i}$ by the corresponding action of a suitable Euler operator, which will automatically generate the correct constant:

$$
\mathcal{Q}[k]=\left(\prod_{i=1}^{k}\left(1+\frac{\underline{u}_{i} \underline{\partial}_{i}}{m+2 \mathbb{E}_{i}-2 i}\right)\right) \underline{\partial}_{x} .
$$

Nevertheless the notation $\mathcal{Q}[k]$ is kept, referring to the length of the highest weight vector, i.e. the number of non-trivial entries $l_{j}$ in its primed notation.

\section{Remark}

In addition to the theory of generalized gradients, one can also obtain this higher spin Dirac operator by means of a suitable projection of the twisted Dirac operator $\underline{\partial}_{x}^{T}: \mathcal{H}_{l_{1}, \cdots, l_{k}} \otimes \mathbb{S} \rightarrow \mathcal{H}_{l_{1}, \cdots, l_{k}} \otimes \mathbb{S}$. It is easily seen, using Klymik's theorem, that this tensor product of $\operatorname{Spin}(m)$-modules contains the module $\mathcal{S}_{l_{1}, \cdots, l_{k}}$, together with (at most) $2^{k}-1$ other irreducible summands. This means that the higher spin Dirac operator can be defined as $\mathcal{Q}_{l_{1}, \ldots, l_{k}}:=\pi_{l_{1}, \cdots, l_{k}}\left[\underline{\partial}_{x}^{T}\right]$, with $\pi_{l_{1}, \cdots, l_{k}}$ the projection operator acting as

$$
\mathcal{H}_{l_{1}, \cdots, l_{k}} \otimes \mathbb{S} \stackrel{\pi_{l_{1}, \cdots, l_{k}}}{\longrightarrow} \mathcal{S}_{l_{1}, \cdots, l_{k}}
$$

Note that this projection operator is different from the one appearing in the application of the Stein-Weiss method. Finally, it should be noted that this definition can also be rewritten in such a way that an inductive structure is revealed:

$$
\mathcal{Q}_{l_{1}, \ldots, l_{k}}=\mathcal{Q}_{l_{1}, \cdots, l_{k-1}}-2 c_{k} \pi_{l_{1}, \cdots, l_{k-1}}\left[\underline{u}_{k}\right]\left\langle\underline{\partial}_{k}, \underline{\partial}_{x}^{T}\right\rangle
$$

In a forthcoming paper we will use this fact to describe general higher spin Dirac operators in terms of twisted higher spin Dirac operators.

\section{Type A solutions of higher spin operators}

As in any function theory, the study of polynomial solutions of the involved differential operators plays a crucial role, due to the fact that these are often used to decompose arbitrary solutions belonging to appropriate $L_{2}$-spaces. We will therefore take a closer look at the homogeneous polynomial solutions of the higher spin Dirac operator (1). 
More specifically, and similarly to what has been done for the Rarita-Schwinger operator and its generalizations, we will study so-called "type A solutions". Indeed, in general two types of homogeneous polynomial solutions of higher spin Dirac operators are to be distinguished: either the polynomial belongs to the kernel of the (twisted) Dirac operator $\underline{\partial}_{x}$, or the operator $\underline{\partial}_{x}$ essentially maps its values to one of the other summands inside the tensor product $\mathcal{H}_{l_{1}, \cdots, l_{k}} \otimes \mathbb{S}$, meaning that the projection operator $\pi_{l_{1}, \cdots, l_{k}}$ then acts trivially. The latter are the so-called "type B solutions", which can be characterized in terms of twistor operators (see e.g. [8] for the case of the Rarita-Schwinger operator). Type A solutions are then obviously polynomials in the kernel of $\underline{\partial}_{x}$ as well as in the respective kernels of the operators $\underline{\partial}_{1}, \ldots, \underline{\partial}_{k}$; hence they must belong to the space $\mathcal{M}_{l_{0}, \ldots, l_{k}}$ defined earlier, where $l_{0}$ now is an additional degree of homogeneity in $\underline{x} \equiv \underline{u}_{0}$. However, in order to ensure that these solutions have the correct values (i.e., simplicial monogenics), we have to consider the following subspace.

Definition 4 For all $(k+1)$-tuples of integers $\left(l_{0}, \ldots, l_{k}\right) \in \mathbb{N}^{k+1}$ satisfying the dominant weight condition $l_{0} \geq \cdots \geq l_{k}$, we define the vector space

$$
\mathcal{M}_{l_{0}, \ldots, l_{k}}^{s}:=\left\{M \in \mathcal{M}_{l_{0}, \ldots, l_{k}}:\left\langle\underline{u}_{1}, \underline{\partial}_{2}\right\rangle M=\cdots=\left\langle\underline{u}_{k-1}, \underline{\partial}_{k}\right\rangle M=0\right\}
$$

The space $\mathcal{M}_{l_{0}, \ldots, l_{k}}^{s}$ now exactly corresponds to the type A solutions of the higher spin Dirac operator $\mathcal{Q}_{l_{1}, \ldots, l_{k}}$. We will study the algebraic structure of this vector space by investigating how it decomposes into irreducible modules for the spin group, and which invariant operators can be used to move between different summands inside this $\operatorname{Spin}(m)$-decomposition. As we will explain in the last section, this question is then related to the topic of transvector algebras, see the monograph [13] and all references mentioned therein.

First of all, let us define the standard general Lie algebra $\mathfrak{g l}_{k}$ (with $k \geq 2$ ), spanned by the standard basis elements $E_{i j}, 1 \leq i, j \leq k$, i.e. the matrices in $\mathbb{C}^{n \times n}$ for which $\left(E_{i j}\right)_{k l}=\delta_{i k} \delta_{j l}$. Finite-dimensional irreducible representations for $\mathfrak{g l}_{k}$ are in one-to-one correspondance with $k$-tuples $\left(\lambda_{1}, \ldots, \lambda_{k}\right) \in \mathbb{C}^{k}$ such that $\lambda_{i}-\lambda_{i+1} \in \mathbb{Z}^{+}$. This $k$-tuple is the highest weight (HW) of the corresponding representation, which we shall denote by $\mathbb{V}(\lambda)$. This module contains, up to a multiple, a unique highest weight vector (HWV) $\underline{v}_{\lambda}$ such that $E_{i i} \underline{v}_{\lambda}=\lambda_{i} \underline{v}_{\lambda}$ and $E_{i j} \underline{v}_{\lambda}=0$ for $i<j$. The reason why we mention these results is the fact that there exists a nice isomorphism between the matrices $E_{i j}$ and the (skew) Euler operators from Clifford analysis, namely $E_{i i} \mapsto \mathbb{E}_{i}+\frac{m}{2}$ and $E_{i j} \mapsto\left\langle\underline{u}_{i}, \underline{\partial}_{j}\right\rangle$, for all $i, j=1, \ldots, k$ and $i \neq j$. For $k=3$, this explicitly yields

$$
\left(\begin{array}{ccc}
E_{11} & E_{12} & E_{13} \\
E_{21} & E_{22} & E_{23} \\
E_{31} & E_{32} & E_{33}
\end{array}\right) \rightarrow\left(\begin{array}{ccc}
\mathbb{E}_{1}+\frac{m}{2} & \left\langle\underline{u}_{1}, \underline{\partial}_{2}\right\rangle & \left\langle\underline{u}_{1}, \underline{\partial}_{3}\right\rangle \\
\left\langle\underline{u}_{3}, \underline{\partial}_{1}\right\rangle & \mathbb{E}_{2}+\frac{m}{2} & \left\langle\underline{u}_{2}, \underline{\partial}_{3}\right\rangle \\
\left\langle\underline{u}_{3}, \underline{\partial}_{1}\right\rangle & \left\langle\underline{u}_{3}, \underline{\partial}_{2}\right\rangle & \mathbb{E}_{3}+\frac{m}{2}
\end{array}\right)
$$

Now, note that the vector space $\mathcal{S}_{l_{1}, \ldots, l_{k}}$ satisfies, as a whole, the conditions for a HWV (by this we mean that, technically speaking, we have as many copies as the dimension of this vector space). In other words, $\mathcal{S}_{l_{1}, \ldots, l_{k}}$ generates a $\mathfrak{g l}_{k}$-module under the action of 
the negative root vectors, given by

$$
\mathbb{V}\left(l_{1}, \ldots, l_{k}\right)^{*} \cong\left(l_{1}+\frac{m}{2}, \cdots, l_{k}+\frac{m}{2}\right)
$$

The upper index * is a shorthand notation for the shift of the HW over half the dimension, and will frequently be used in what follows. The following can then easily be proven, using the fact that $\left[\underline{\partial}_{i}, E_{p q}\right]=\delta_{i p} \underline{\partial}_{q}$ (for $i \neq j$ ).

Lemma 1 Each element $E_{i j}$ of the algebra $\mathfrak{g l}_{k}$ acts as an endomorphism on the (total) space of monogenic polynomials in several variables.

In other words, each spinor-valued polynomial of the form

$$
\left[\sum_{\left(p_{i j}\right)}\left(\prod_{i, j} E_{i j}^{p_{i j}}\right)\right] S\left(\underline{u}_{1}, \cdots, \underline{u}_{k}\right), \quad p_{i j} \in \mathbb{N}
$$

is still monogenic in several variables. Here $S\left(\underline{u}_{1}, \ldots, \underline{u}_{k}\right)$ is simplicial monogenic and the factor between brackets denotes an arbitrary word in the (skew) Euler operators generating $\mathfrak{g l}_{k}$. This can also be formulated in the following way.

Lemma 2 The elements of the universal enveloping algebra $U\left(\mathfrak{g l}_{k}\right)$ preserve the (total) space of monogenic polynomials in $k$ vector variables.

Moreover, no other words have this property, which is a crucial observation. To explain what this means, we need the following auxiliary result.

Lemma 3 The vector variables $\left\{\underline{u}_{i}\right\}_{i=1}^{k}$ and their corresponding Dirac operators $\left\{\underline{\partial}_{i}\right\}_{i=1}^{k}$ generate a model for the Lie superalgebra $\mathfrak{o s p}(1,2 k)$.

This can be proven by direct calculation, invoking rather well-known operator identities between Clifford operators. When decomposing polynomial vector spaces in $k$ vector variables in terms of irreducible modules for the spin group, one needs two pieces of information: highest weights, referring to which summands to include, and the socalled embedding factors, referring to how to include these summands. For example, it is well-known that the space of $\mathbb{S}$-valued harmonics of degree $k$ decomposes as $\mathcal{H}\left(\mathbb{R}^{m}, \mathbb{S}\right)=\mathcal{M}_{k} \oplus \underline{x} \mathcal{M}_{k-1}$, and in this example the variable $\underline{x}$ plays the role of embedding factor (as a multiplication operator). Obviously the situation of considering $k$ vector variables is similar, the only difference being that there are more possibilities for the choice of the embedding factors. Since these factors have to be polynomial invariants, we can easily list all those possibilities: they precisely correspond to products of elements in the algebra $\mathfrak{o s p}(1,2 k)$, i.e. elements in the algebra $\mathrm{U}(\mathfrak{o s p}(1,2 k))$. Next, the wellknown PBW-theorem tells us that we can always rearrange these products according to a chosen ordering. Choosing the ordering on the generators of $\mathfrak{o s p}(1,2 k)$ such that

(i) first all combinations involving the vector variables only are listed

(ii) then all elements in $\mathfrak{g l}_{k}$ are listed

(iii) finally all combinations involving Dirac operators only are listed, 
it follows that the only elements in the universal enveloping algebra $\mathrm{U}(\mathfrak{o s p}(1,2 k))$ which can be used as embedding factors, are elements in $\mathrm{U}\left(\mathfrak{g l}_{k}\right)$. Indeed: combinations involving type (iii) will always act trivially on the space of simplicial monogenics, whereas combinations involving type (i) will always belong to the Fischer complement of the space of monogenic polynomials. The latter statement is based on the fact that

$$
\mathcal{P}\left(\mathbb{R}^{k m}, \mathbb{S}\right)=\mathcal{M}\left(\mathbb{R}^{k m}, \mathbb{S}\right) \oplus\left(\underline{u}_{1} \mathcal{P}\left(\mathbb{R}^{k m}, \mathbb{S}\right)+\cdots+\underline{u}_{k} \mathcal{P}\left(\mathbb{R}^{k m}, \mathbb{S}\right)\right)
$$

the sum between brackets obviously not being direct. We are then lead to the following important conclusion.

Proposition 1 In order to decompose the polynomial vector space $\mathcal{M}_{l_{1}, \cdots, l_{k}}$ into irreducible modules for the spin group, it suffices to select all weight spaces having the correct degree of homogeneity inside each of the $\mathfrak{g l}_{k}$-modules $\mathbb{V}\left(\lambda_{1}, \cdots, \lambda_{k}\right)^{*}$ generated by the spaces of simplicial monogenics $\mathcal{S}_{\lambda_{1}, \cdots, \lambda_{k}}$.

\section{Example}

Despite the fact that the case $k=2$ is rather trivial, it is still useful to illustrate the procedure described above. Suppose that we want to decompose the vector space $\mathcal{M}_{l_{1}, l_{2}}$, $l_{1} \geq l_{2}$. We then need to consider the $\mathfrak{g l}_{2}$-modules generated by the spaces $\mathcal{S}_{p, q}, p \geq q$. The definition of $\mathcal{S}_{p, q}$ yields

$$
\mathbb{V}(p, q)^{*}=\mathcal{S}_{p, q} \oplus\left\langle\underline{u}_{2}, \underline{\partial}_{1}\right\rangle \mathcal{S}_{p, q} \oplus \cdots \oplus\left\langle\underline{u}_{2}, \underline{\partial}_{1}\right\rangle^{p-q} \mathcal{S}_{p, q}
$$

where it is easily verified that only a limited number of these modules will contribute to the space $\mathcal{M}_{l_{1}, l_{2}}$. Selecting the ones showing the correct degree of homogeneity, we thus indeed have that

$$
\mathcal{M}_{l_{1}, l_{2}}=\mathcal{S}_{l_{1}, l_{2}} \oplus\left\langle\underline{u}_{2}, \underline{\partial}_{1}\right\rangle \mathcal{S}_{l_{1}+1, l_{2}-1} \oplus \cdots \oplus\left\langle\underline{u}_{2}, \underline{\partial}_{1}\right\rangle^{l_{2}} \mathcal{S}_{l_{1}+l_{2}, 0}
$$

This result was already obtained in e.g. [8].

In the general case, the procedure becomes more complicated since the weight spaces in arbitrary $\mathfrak{g l}_{k}$-modules (with $k>2$ ) occur with higher multiplicity, meaning that also the decomposition for $\mathcal{M}_{l_{1}, \cdots, l_{k}}$ will no longer be multiplicity-free.

As a direct consequence of Proposition 1, techniques from representation theory can be used for $\mathfrak{g l}_{k+1}$ in order to obtain results on the space $\mathcal{M}_{l_{0}, \ldots, l_{k}}^{s}$ containing $\left(l_{0^{-}}\right.$ homogeneous) type A solutions of the higher spin Dirac operator $\mathcal{Q}_{l_{1}, \cdots, l_{k}}$. However we should take into account that not all $\mathbb{S}$-valued polynomials within the module $\mathbb{V}\left(l_{0}, \ldots, l_{k}\right)^{*}$ can be seen as type A solutions of $\mathcal{Q}_{l_{1}, \cdots, l_{k}}$, since only a specific subspace of it will show the right values. Hence, we still have to intersect the space of monogenics in several variables with the respective kernels of the operators $E_{i j}$, where $1<i<j \leq k+1$. Here we need to add a remark on the notations: as we have included the additional vector variable $\underline{x}$, formally denoted as $\underline{u}_{0}$ (and $\underline{\partial}_{x}$ as $\underline{\partial}_{0}$ ), the isomorphism between the matrices $E_{i j}$ and the (skew) Euler operators has shifted to

$$
E_{i i} \mapsto \mathbb{E}_{i-1}+\frac{m}{2}, \quad E_{i j} \mapsto\left\langle\underline{u}_{i-1}, \underline{\partial}_{j-1}\right\rangle, \quad i, j=1, \ldots, k+1, i \neq j
$$


Note that we thus needed to exclude $E_{12}$ (corresponding to $\left\langle\underline{x}, \underline{\partial}_{1}\right\rangle$ ) from the intersection mentioned above, otherwise we would only retrieve spaces of simplicial monogenics as a result. So, not all polynomials of the form (3) will contribute to the space $\mathcal{M}_{l_{0}, \ldots, l_{k}}^{s}$ : certain restrictions have to be imposed. In order to describe these restrictions, it suffices to realize that the desired polynomials should satisfy the conditions to be a HWV for the algebra $\mathfrak{g l}_{k}$, whence the language of branching may be used. To this end, we define the subspace $\mathbb{V}(\lambda)^{+}$of $\mathbb{V}(\lambda) \equiv \mathbb{V}\left(\lambda_{0}, \ldots, \lambda_{k}\right)$, containing all HWV of the subalgebra $\mathfrak{g l}_{k} \subset \mathfrak{g l}_{k+1}$ :

$$
\mathbb{V}(\lambda)^{+}=\left\{\eta \in \mathbb{V}(\lambda): E_{i j} \eta=0,2 \leq i<j \leq k+1\right\}
$$

Moreover, we introduce a notation for the set of weight spaces in $\mathbb{V}(\lambda)$ realizing a copy of the $\mathfrak{g l}_{k}$-module with highest weight $\mu=\left(\mu_{1}, \ldots, \mu_{k}\right)$. This means that for each of the elements in the previous set, a subscript $\mu$ is added referring to the $\mathfrak{g l}_{k}$-module for which it actually defines a HWV, viz

$$
\mathbb{V}(\lambda)_{\mu}^{+}=\left\{\eta \in \mathbb{V}(\lambda)^{+}: E_{i i} \eta=\mu_{i-1} \eta, 2 \leq i \leq k+1\right\}
$$

As $\mathbb{V}(\lambda)$ is generated by the operators $E_{i j}$ acting on the space $\mathcal{S}_{\lambda}$, each element $\eta \in \mathbb{V}(\lambda)_{\mu}^{+}$ is to be seen as a particular element of the form $(3)$, with $S\left(\underline{x}, \underline{u}_{1}, \ldots, \underline{u}_{k}\right) \in \mathcal{S}_{\lambda}$. Recall that the dimension of the spaces $\mathbb{V}(\lambda)_{\mu}^{+}$is either 0 or 1 , with

$$
\operatorname{dim}\left(\mathbb{V}(\lambda)_{\mu}^{+}\right)=1 \Leftrightarrow \lambda_{i-1}-\mu_{i} \in \mathbb{Z}^{+} \text {and } \mu_{i}-\lambda_{i} \in \mathbb{Z}^{+}, \text {for all } i=1, \ldots, k
$$

which is called the "betweenness" condition, as it can be represented graphically -at least for integer values of $\lambda_{i}$ or integer values shifted over half the dimension- by

$$
\lambda_{0} \geq \mu_{1} \geq \lambda_{1} \geq \mu_{2} \geq \lambda_{2} \geq \cdots \geq \lambda_{k-1} \geq \mu_{k} \geq \lambda_{k}
$$

In order to explain how this restricts the number of summands which can contribute to the space of type A solutions of a higher spin Dirac operator, let us consider an illustrative example with $k=2$ and $\left(\lambda_{0}, \lambda_{1}, \lambda_{2}\right)=(4,3,1)^{*}$. According to the branching rules, when considering $\mathbb{V}(\lambda)$ as a $\mathfrak{g l}_{2}$-module, only the following summands survive:

$$
\left.\mathbb{V}(4,3,1)^{*}\right|_{\mathfrak{g l}_{2}} ^{\mathfrak{g l}_{3}} \cong\left((4,3)^{*} \oplus(4,2)^{*} \oplus(4,1)^{*}\right) \oplus\left((3,3)^{*} \oplus(3,2)^{*} \oplus(3,1)^{*}\right)
$$

In the above expression each of the terms between brackets stands for a combination of the following form, written in terms of the negative root vectors for $\mathfrak{g l}_{3}$ :

$$
\left(\sum_{a, b, c} E_{21}^{a} E_{31}^{b} E_{32}^{c}\right) \mathcal{S}_{4,3,1}
$$

Moreover, the result should still belong to $\operatorname{ker}\left(E_{23}\right)$, with $E_{23}$ the unique positive root vector characterizing the algebra $\mathfrak{g l}_{2} \subset \mathfrak{g l}_{3}$. The algebra $\mathfrak{g l}_{2}$ has Cartan elements $E_{22}$ and $E_{33}$, meaning that the six couples of integers above are in fact the degrees of homogeneity in $\left(\underline{u}_{1}, \underline{u}_{2}\right)$. In this way limitations on the degree of homogeneity of the embedding factors are obtained. Moreover, looking at the summands above (or at the 
betweenness condition for the most general case), it is clear that none of the embedding factors will have an effect of the form $( \pm 1, \mp 1)$ on the degree in $\left(\underline{u}_{1}, \underline{u}_{2}\right)$. So there is no need to include the factor $E_{32}$, which corresponds to the final result having to be in $\operatorname{ker}\left(E_{23}\right)$. As we will see in the next section, this statement is not yet precise: we will prove that $E_{32}$ can occur, but taking into account homogeneities, the embedding factor as a whole will always behave as the term $E_{21}^{a} E_{31}^{b}$, which, in some sense, is the leading term. For example, in order to have that $\left(E_{21}^{a} E_{31}^{b}\right) \mathcal{S}_{4,3,1} \leftrightarrow(4,2)$ we must have that $(3+a, 1+b)=(4,2)$, or $(a, b)=(1,1)$. In other words: the branching rules tell us which degrees of homogeneity to expect for the (leading term in the) embedding factors.

We may now formulate the following general result.

Proposition 2 For each vector space $\mathcal{S}_{\lambda_{0}, \cdots, \lambda_{k}}$, the only summands inside the $\mathfrak{g l}_{k+1^{-}}$module $\mathbb{V}\left(\lambda_{0}, \cdots, \lambda_{k}\right)^{*}$ contributing to the space of type $A$ solutions of the higher spin Dirac operator in $k$ dummy vector variables are of the form

$$
\rho_{d_{1}, \cdots, d_{k}} \mathcal{S}_{\lambda_{0}, \cdots, \lambda_{k}}
$$

where $\rho_{d_{1}, \cdots, d_{k}} \in U\left(\mathfrak{g l}_{k+1}\right)$ is an embedding factor which is homogeneous of degree $\left(d_{1}, \cdots, d_{k}\right)$ in $\left(\underline{u}_{1}, \cdots, \underline{u}_{k}\right)$. Moreover, the integers $d_{j}$ satisfy the following conditions:

$$
\lambda_{0} \geq \lambda_{1}+d_{1} \geq \lambda_{1} \geq \lambda_{2}+d_{2} \geq \cdots \geq \lambda_{k-1} \geq \lambda_{k}+d_{k} \geq \lambda_{k}
$$

or $0 \leq d_{p} \leq \lambda_{p-1}-\lambda_{p}$ (with $\left.1 \leq p \leq k\right)$.

In the next section, an explicit form for these embedding factors $\rho_{d_{1}, \cdots, d_{k}}$ is obtained, using results on raising and lowering operators in transvector algebras. Note that these factors will be unique up to a constant, which follows from the fact that the branching from $\mathfrak{g l}_{k+1}$ to $\mathfrak{g l}_{k}$ is multiplicity-free.

\section{Example}

Suppose we want to describe the space $\mathcal{M}_{3,1,1}^{s}$, i.e. the space of 3-homogeneous type A solutions of the operator $\mathcal{Q}_{1,1}$, studied in [14]. This is the invariant operator acting on spinor-valued forms, see also [15]. Hence we are looking for 3-tuples of integers $\left(\lambda_{0}, \lambda_{1}, \lambda_{2}\right)^{*}$ such that $\rho_{a, b} \mathcal{S}_{\lambda_{0}, \lambda_{1}, \lambda_{2}} \subset \mathcal{M}_{3,1,1}^{s}$, meaning that the following conditions have to be satisfied:

$$
\left(\lambda_{0}-a-b, \lambda_{1}+a, \lambda_{2}+b\right)=(3,1,1) \text { and }\left\{\begin{array}{l}
\lambda_{0}-\lambda_{1} \geq a \geq 0 \\
\lambda_{1}-\lambda_{2} \geq b \geq 0
\end{array}\right.
$$

Now, obviously $(a, b)=(0,0)$ leads to the summand $\mathcal{S}_{3,1,1} \subset \mathcal{M}_{3,1,1}^{s}$, as was to be expected, since, in general, the solution $d_{1}=\cdots=d_{k}=0$ will always be there. Any other solution is non-trivial, which means that $\lambda_{0}>3$. As $\lambda_{1} \geq \lambda_{2}$, the only other possibility is $(a, b)=(0,1)$. Note that $\left(\lambda_{0}, \lambda_{1}, \lambda_{2}\right)=(5,0,0)$ is not allowed, as follows from the condition on $b$. This means that $\mathcal{M}_{3,1,1}^{s} \cong \mathcal{S}_{3,1,1} \oplus \mathcal{S}_{4,1}$, which corresponds to the results of [14].

Let us now formulate the main conclusion of this section. 
Theorem 2 As a module for the spin group, the space $\mathcal{M}_{l_{0}, \cdots, l_{k}}^{s}$ decomposes into the following irreducible summands:

$$
\mathcal{M}_{l_{0}, \cdots, l_{k}}^{s}=\bigoplus_{\left(d_{1}, \cdots, d_{k}\right)} \rho_{d_{1}, \cdots, d_{k}} \mathcal{S}_{\lambda_{0}, \cdots, \lambda_{k}}
$$

where $\left(\lambda_{0}, \cdots, \lambda_{k}\right)^{\prime}$ is a dominant weight satisfying

$$
\left(\lambda_{0}, \lambda_{1}, \cdots, \lambda_{k}\right)=\left(l_{0}+\sum_{i=1}^{k} d_{i}, l_{1}-d_{1}, \cdots, l_{k}-d_{k}\right)
$$

with $l_{i}-l_{i+1} \geq d_{i} \geq 0$ for $1 \leq i \leq k-1$ and $0 \leq d_{k} \leq l_{k}$. At the same time, this is the decomposition of the space of $l_{0}$-homogeneous type $A$ solutions of the operator $\mathcal{Q}_{l_{1}, \cdots, l_{k}}$.

Proof

First, it follows from the branching rules for $\mathfrak{g l}_{k+1}$ to $\mathfrak{g l}_{k}$ that no embedding factor $\rho_{d_{1}, \cdots, d_{k}}$ can have a net effect of the form $( \pm p, \mp p)$ on the homogeneity degree in two variables $\left(\underline{u}_{i}, \underline{u}_{j}\right)$, with $i, j \geq 1$ and $p \in \mathbb{N}$. Indeed:

$$
\left.\left(\lambda_{2}, \cdots, \lambda_{k}\right)^{\prime} \subset\left(\lambda_{1}, \cdots, \lambda_{k}\right)^{\prime}\right|_{\mathfrak{g l}_{k}} ^{\mathfrak{g l}_{k+1}}
$$

and any other summand which comes from the branching is obtained by adding positive integers. This implies that the net effect of the factor $\rho_{d_{1}, \cdots, d_{k}}$ can always be represented with a leading term of the form $\rho_{d_{1}, \cdots, d_{k}}=E_{21}^{d_{1}} \cdots E_{(k+1) 1}^{d_{k}}+\cdots$, where the numbers $\left(d_{1}, \cdots, d_{k}\right)$ satisfy the betweenness conditions coming from the branching. If we then fix the numbers $\left(l_{0}, \cdots, l_{k}\right)$, it suffices to find all the $(k+1)$-tuples $\left(\lambda_{0}, \cdots, \lambda_{k}\right)$ for which there exist positive integers $d_{j}$ such that we have an inclusion $\rho_{d_{1}, \cdots, d_{k}} \mathcal{S}_{\lambda_{0}, \cdots, \lambda_{k}} \subset \mathcal{M}_{l_{0}, \cdots, l_{k}}$. This is only possible if the conditions

$$
\left(\lambda_{0}-\sum_{i=1}^{k} d_{i}, \lambda_{1}+d_{1}, \cdots, \lambda_{k}+d_{k}\right)=\left(l_{0}, \cdots, l_{k}\right)
$$

on the degrees of homogeneity are satisfied, and if moreover

$$
\left\{\begin{array}{c}
\lambda_{0}-\lambda_{1} \geq d_{1} \geq 0 \\
\lambda_{1}-\lambda_{2} \geq d_{2} \geq 0 \\
\vdots \\
\lambda_{k-1}-\lambda_{k} \geq d_{k} \geq 0
\end{array}\right.
$$

These are the conditions coming from the branching rules. Using the restrictions on the homogeneity, this can also be rewritten as $l_{i}-l_{i+1} \geq d_{i} \geq 0$, for all $1 \leq i<k$, and $\lambda_{k}=l_{k}-d_{k}$. This last equation tells us that $0 \leq d_{k} \leq l_{k}$. 


\section{Relation with transvector algebras}

The aim of this section is to obtain explicit expressions for the embedding factors $\rho_{d_{1}, \cdots, d_{k}}$, i.e. the elements in $\mathrm{U}\left(\mathfrak{g l}_{k+1}\right)$ realizing the decomposition of the space $\mathcal{M}_{l_{0}, \cdots, l_{k}}^{s}$ into irreducible summands under the spin group.

To this end, we first introduce the elements $z_{i 1}$ and $z_{1 i}, i=2, \ldots k+1$, in the universal enveloping algebra $\mathrm{U}\left(\mathfrak{g l}_{k+1}\right)$ :

$$
\begin{aligned}
z_{i 1} & =\sum_{i>i_{1}>\cdots>i_{s}>1} E_{i i_{1}} E_{i_{1} i_{2}} \cdots E_{i_{s-1} i_{s}} E_{i_{s} 1}\left(h_{i}-h_{j_{1}}\right) \cdots\left(h_{i}-h_{j_{r}}\right) \\
z_{1 i} & =\sum_{i<i_{1}<\cdots<i_{s} \leq k+1} E_{i_{1} i} E_{i_{2} i_{1}} \cdots E_{i_{s} i_{s-1}} E_{1 i_{s}}\left(h_{i}-h_{j_{1}}\right) \cdots\left(h_{i}-h_{j_{r}}\right)
\end{aligned}
$$

In these definitions, $s$ runs over nonnegative integers, $h_{i}=E_{i i}-i+1$ and $\left\{j_{1}, \ldots, j_{r}\right\}$ is the complementary subset to $\left\{i_{1}, \ldots, i_{s}\right\}$ in the set $\{1, \ldots i-1\}$ or $\{i+1, \ldots, k+1\}$. For example, when $k=3$ we have that

$$
z_{41}=E_{41}\left(h_{4}-h_{2}\right)\left(h_{4}-h_{3}\right)+E_{43} E_{31}\left(h_{4}-h_{2}\right)+E_{42} E_{21}\left(h_{4}-h_{3}\right)+E_{43} E_{32} E_{21}
$$

Although this definition seems rather ad hoc, one can actually construct these operators using the language of extremal projection operators, see e.g. $[13,16]$ and the references mentioned therein. These operators have the following property.

Lemma 4 Let $\eta \in \mathbb{V}(\lambda)_{\mu}^{+}, \mu=\left(\mu_{1}, \ldots, \mu_{k}\right)$. Then, for any $i=2, \ldots, k+1$, we have

$$
z_{i 1} \eta \in \mathbb{V}(\lambda)_{\mu+\delta_{i-1}}^{+}, \quad z_{1 i} \eta \in \mathbb{V}(\lambda)_{\mu-\delta_{i-1}}^{+}
$$

where the weight $\mu \pm \delta_{i-1}$ is obtained from $\mu$ by replacing $\mu_{i-1}$ by $\mu_{i-1} \pm 1$.

This was proven in [16]. In the present setting of solutions of higher spin operators, the lemma can be reformulated as: the operators $z_{i 1}$ and $z_{1 i}, i=2, \ldots, k+1$ will map a type A solution of a higher spin Dirac operator to another type A solution (be it for another operator, since the degree of homogeneity will change). More explicitly, the following results hold.

Corollary 1 For every polynomial $P\left(\underline{x} ; \underline{u}_{1}, \cdots, \underline{u}_{k}\right) \in \mathcal{M}_{l_{0}, \cdots, l_{k}}^{s}$, we have

$$
\begin{aligned}
& z_{i 1} P\left(\underline{x} ; \underline{u}_{1}, \cdots, \underline{u}_{k}\right) \in \mathcal{M}_{l_{0}-1, l_{1}, \cdots, l_{i-2}, l_{i-1}+1, l_{i}, \cdots, l_{k}}^{s} \\
& z_{1 i} P\left(\underline{x} ; \underline{u}_{1}, \cdots, \underline{u}_{k}\right) \in \mathcal{M}_{l_{0}+1, l_{1}, \cdots, l_{i-2}, l_{i-1}-1, l_{i}, \cdots, l_{k}}^{s}
\end{aligned}
$$

\section{Example}

When $k=2$, we have that

$$
\begin{array}{ll}
z_{21}=E_{21} & =\left\langle\underline{u}_{1}, \underline{\partial}_{x}\right\rangle \\
z_{31}=E_{32} E_{21}+E_{31}\left(h_{3}-h_{2}\right) & =\left\langle\underline{u}_{2}, \underline{\partial}_{1}\right\rangle\left\langle\underline{u}_{1}, \underline{\partial}_{x}\right\rangle+\left\langle\underline{u}_{2}, \underline{\partial}_{x}\right\rangle\left(\mathbb{E}_{2}-\mathbb{E}_{1}-1\right) \\
z_{12}=E_{32} E_{13}+E_{12}\left(h_{2}-h_{3}\right) & =\left\langle\underline{u}_{2}, \underline{\partial}_{1}\right\rangle\left\langle\underline{x}, \underline{\partial}_{2}\right\rangle+\left\langle\underline{x}, \underline{\partial}_{1}\right\rangle\left(\mathbb{E}_{1}-\mathbb{E}_{2}+1\right) \\
z_{13}=E_{13} & =\left\langle\underline{x}, \underline{\partial}_{2}\right\rangle .
\end{array}
$$


In view of Lemma $4, z_{31}$ raises the degree in $\underline{u}_{2}$ by one. More explicitly, this means that the operator $z_{31}$ maps $l_{0}$-homogeneous solutions of the operator $\mathcal{Q}_{l_{1}, l_{2}}$ to $\left(l_{0}-1\right)$ homogeneous solutions of the operator $\mathcal{Q}_{l_{1}, l_{2}+1}$. Reconsidering the space $\mathcal{M}_{3,1}^{s}$, we can now write its direct sum decomposition in terms of the explicit embedding factors:

$$
\mathcal{M}_{3,1,1}^{s}=\mathcal{S}_{3,1,1} \oplus\left(\left\langle\underline{u}_{2}, \underline{\partial}_{1}\right\rangle\left\langle\underline{u}_{1}, \underline{\partial}_{x}\right\rangle+\left\langle\underline{u}_{2}, \underline{\partial}_{x}\right\rangle\left(\mathbb{E}_{2}-\mathbb{E}_{1}-1\right)\right) \mathcal{S}_{4,1}
$$

Note that the Euler operators will only produce multiplicative constants, since they act on homogeneous polynomials. In this way, we also see the aforementioned leading terms in the example, up to a constant.

In order to explain the relation to the theory of transvector algebras, a few more concepts have to be introduced. First, consider once more the universal enveloping algebra $\mathrm{U}\left(\mathfrak{g l}_{k+1}\right)$ of $\mathfrak{g l}_{k+1}$, as well as the direct sum decomposition $\mathfrak{g l}_{k}=\mathfrak{g l}_{k}^{-} \oplus \mathfrak{h} \oplus \mathfrak{g l}_{k}^{+}$ of the subalgebra $\mathfrak{g l}_{k}$, where $\mathfrak{g l}_{k}^{ \pm}$denotes the space of positive and negative root vectors, and $\mathfrak{h}$ is the Cartan algebra. Let $R(\mathfrak{h})$ be the field of fractions of $\mathfrak{h}$, then the natural extension $\mathrm{U}^{\prime}\left(\mathfrak{g l}_{k+1}\right):=\mathrm{U}\left(\mathfrak{g l}_{k+1}\right) \otimes_{U(\mathfrak{h})} R(\mathfrak{h})$ of $\mathrm{U}\left(\mathfrak{g l}_{k+1}\right)$ provides a means of dividing by Euler operators, since the field of fractions exactly consists of all rational operators whose nominator and denominator are linear combinations of the $E_{i i}$. Next, consider the left ideal $J^{\prime}:=\mathrm{U}^{\prime}\left(\mathfrak{g l}_{k+1}\right) \mathfrak{g l}_{k}^{+} \subset \mathrm{U}^{\prime}\left(\mathfrak{g l}_{k+1}\right)$, as well as its normalizer $\operatorname{Norm}\left(J^{\prime}\right):=\left\{u \in \mathrm{U}^{\prime}\left(\mathfrak{g l}_{k+1}\right): J^{\prime} u \in J^{\prime}\right\} . \quad J^{\prime}$ being a twosided ideal in $\operatorname{Norm}\left(J^{\prime}\right)$, one finally can define

$$
Z\left(\mathfrak{g l}_{k+1}, \mathfrak{g l}_{k}\right):=\operatorname{Norm}\left(J^{\prime}\right) / J^{\prime}
$$

In the literature this quotient is known as the Mickelsson-Zhelobenko algebra. As was shown e.g. in [13], the elements $z_{i 1}$ and $z_{1 i}, i=2, \ldots, k+1$, form a set of generators of this Mickelsson algebra $Z\left(\mathfrak{g l}_{k+1}, \mathfrak{g l}_{k}\right)$.

Lemma 5 Let $\mu$ satisfy the betweenness condition stated above, and let $\underline{v}_{\lambda}$ be the highest weight vector of the module $\mathbb{V}(\lambda)$. Then the elements

$$
\underline{v}_{\lambda}(\mu):=z_{21}^{d_{1}} \cdots z_{(k+1) 1}^{d_{k}} \underline{v}_{\lambda}
$$

are nonzero, provided that $\left(d_{1}, \cdots, d_{k}\right)$ satisfies all conditions of Theorem 2. Moreover, the space $\mathbb{V}(\lambda)^{+}$is spanned by these elements $\underline{v}_{\lambda}(\mu)$.

\section{Example}

As before, take $k=2$ and $\lambda=(4,3,1)^{*}$, and consider the module $\mathbb{V}(4,3,1)^{*}$ generated by the space $\mathcal{S}_{4,3,1}$. Lemma 5 then states that consecutive actions of the operators $z_{21}$ and $z_{31}$ will produce a basis of the space $\mathbb{V}(4,3,1)^{*} \cap \operatorname{ker}\left\langle\underline{u}_{1}, \underline{\partial}_{2}\right\rangle$. More precisely, we obtain the following spaces, corresponding to the 6 possible choices for $\mu$, and the respective spaces of higher spin solutions to which they contribute, see (4):

$$
\begin{array}{l|l}
\mathcal{S}_{4,3,1} & \mathcal{M}^{s}(4,3,1) \\
z_{21} \mathcal{S}_{4,3,1} & \mathcal{M}^{s}(3,4,1) \\
z_{31} \mathcal{S}_{4,3,1} & \mathcal{M}^{s}(3,3,2) \\
z_{21} z_{31} \mathcal{S}_{4,3,1} & \mathcal{M}^{s}(2,4,2) \\
z_{31}^{2} \mathcal{S}_{4,3,1} & \mathcal{M}^{s}(2,3,3) \\
z_{21} z_{31}^{2} \mathcal{S}_{4,3,1} & \mathcal{M}^{s}(1,4,3)
\end{array}
$$


Note however that this is not the decomposition of $\mathcal{M}_{4,3,1}^{s}$. Indeed, using the correct embedding factors, we get that the latter is equal to

$$
\mathcal{M}_{4,3,1}^{s}=\mathcal{S}_{4,3,1} \oplus z_{21} \mathcal{S}_{5,2,1} \oplus z_{21}^{2} \mathcal{S}_{6,1,1} \oplus z_{31} \mathcal{S}_{5,3,0} \oplus z_{21} z_{31} \mathcal{S}_{6,2,0} \oplus z_{21}^{2} z_{31} \mathcal{S}_{7,1,0}
$$

Recall that the embedding factor, as a whole, should behave as $E_{21}^{a} E_{31}^{b}$, with this term itself as a leading term. This might not be so obvious from the definitions and lemmas stated above. Note though that the operators $z_{i 1}$ actually are defined up to a constant factor, or more precisely: up to an expression in terms of Euler operators. Since we are working within the field of fractions $R(\mathfrak{h})$, it is possible to divide $z_{i 1}$ by $\left(h_{i}-h_{i-1}\right) \ldots\left(h_{i}-h_{2}\right)$, so that the resulting operators $s_{i 1}$ (and likewise $\left.s_{1 i}\right)$ take the form

$$
\begin{aligned}
s_{i 1} & =\sum_{i>i_{1}>\cdots>i_{s}>1} E_{i i_{1}} E_{i_{1} i_{2}} \cdots E_{i_{s-1} i_{s}} E_{i_{s}} \frac{1}{\left(h_{i}-h_{i_{1}}\right) \cdots\left(h_{i}-h_{i_{s}}\right)} \\
s_{1 i} & =\sum_{i<i_{1}<\cdots<i_{s} \leq k+1} E_{i i_{1}} E_{i_{1} i_{2}} \cdots E_{i_{s-1} i_{s}} E_{i_{s} 1} \frac{1}{\left(h_{i}-h_{i_{1}}\right) \cdots\left(h_{i}-h_{i_{s}}\right)}
\end{aligned}
$$

or still $s_{i 1}=E_{i 1}+$ other operators, which proves the statement: it is now easily seen that powers of the operators $s_{i 1}$ or $s_{1 i}$ indeed behave as the leading terms predicted earlier. For instance, after rescaling, the four operators in (5) become

$$
\left\{\begin{aligned}
s_{21} & =\left\langle\underline{u}_{1}, \underline{\partial}_{x}\right\rangle \\
s_{31} & =\left\langle\underline{u}_{2}, \underline{\partial}_{x}\right\rangle+\left\langle\underline{u}_{2}, \underline{\partial}_{1}\right\rangle\left\langle\underline{u}_{1}, \underline{\partial}_{x}\right\rangle \frac{1}{\mathbb{E}_{2}-\mathbb{E}_{1}-1} \\
s_{12} & =\left\langle\underline{x}, \underline{\partial}_{1}\right\rangle+\left\langle\underline{u}_{2}, \underline{\partial}_{1}\right\rangle\left\langle\underline{x}, \underline{\partial}_{2}\right\rangle \frac{1}{\mathbb{E}_{1}-\mathbb{E}_{2}+1} \\
s_{13} & =\left\langle\underline{x}, \underline{\partial}_{2}\right\rangle .
\end{aligned}\right.
$$

So, the embedding factors defined in Proposition 2, and playing a crucial role in Theorem 2 , are given by $\rho_{d_{1}, d_{2}, \cdots, d_{k}}=s_{21}^{d_{1}} \cdots s_{(k+1) 1}^{d_{k}}$. This is a result of Lemma 5 .

\section{References}

[1] Brackx F, Delanghe R and Sommen F 1982, Clifford Analysis, Research Notes in Mathematics 76, Pitman, London.

[2] Delanghe R, Sommen F and Souček V 1992, Clifford analysis and spinor valued functions, Kluwer Academic Publishers, Dordrecht.

[3] Gilbert J and Murray MAM 1991, Clifford algebras and Dirac operators in harmonic analysis, Cambridge University Press, Cambridge.

[4] Delanghe R 2001, Clifford analysis: history and perspective, Comp. Meth. Funct. Theory 1, 107153.

[5] Branson T 1997, Stein-Weiss operators and ellipticity, J. Funct. Anal. 151(2), 334-383.

[6] Fegan HD 1976, Conformally invariant first order differential operators, Quart. J. Math. 27, 513538.

[7] Stein EW and Weiss G 1968, Generalization of the Cauchy-Riemann equations and representations of the rotation group, Amer. J. Math. 90, 163-196.

[8] Bureš J, Sommen F, Souček V and Van Lancker P 2001, Rarita-Schwinger type operators in Clifford analysis, J. Funct. Anal. 185, 425-456. 
[9] Bureš J, Sommen F, Souček V and Van Lancker P 2001, Symmetric analogues of Rarita-Schwinger equations, Ann. Glob. Anal. Geom. 21(3), 215-240.

[10] Constales D, Sommen F and Van Lancker P 2001, Models for irreducible representations of $\operatorname{Spin}(m)$, Adv. Appl. Cliff. Alg. 11(S1), 271-289.

[11] Eelbode D and Van de Voorde L 2010, A toy model for higher spin Dirac operators, Physics of Atomic Nuclei 73(2), 282-287.

[12] Eelbode D and Van de Voorde L 2008, Higher spin operators and polyharmonic functions, Proc. of the 16th ICFIDCAA (Korea), 137-142.

[13] Molev AI 2007, Yangians and classical Lie algebras (Mathematical surveys and monographs 143) (AMS Bookstore).

[14] Bureš J 1999, The higher spin Dirac operators, Differential geometry and applications (Masaryk Univ. Brno), 319-334.

[15] Severa V 1998, Invariant differential operators between spinor-valued forms (PhD-thesis, Charles University, Prague).

[16] Molev AI 2000, Weight bases of Gelfand-Tsetlin type for representations of classical Lie algebras, Journ. Phys. A 33(22), 4143-4158. 